\title{
One-year mortality after hospital admission as an indicator of palliative care need: A retrospective cohort study
}

\author{
P Frankenfeld, ${ }^{1} \mathrm{MB}$ ChB, FCP (SA); L van Niekerk, ${ }^{2} \mathrm{MB}$ ChB, MSc; K Manning, ${ }^{1} \mathrm{MSc}(\mathrm{Med}) ; \mathrm{N}$ Tiffin, ${ }^{3,4} \mathrm{PhD}$; \\ P J Raubenheimer, ${ }^{1} \mathrm{MB}$ BCh, FCP (SA) \\ ${ }^{1}$ Division of General Medicine, Department of Medicine, Groote Schuur Hospital and Faculty of Health Sciences, University of Cape Town, \\ South Africa \\ ${ }^{2}$ Department of Global Health, London School of Hygiene and Tropical Medicine, London, UK \\ ${ }^{3}$ Centre for Infectious Disease Epidemiology Research, School of Public Health and Family Medicine, Faculty of Health Sciences, University of \\ Cape Town, South Africa \\ ${ }^{4}$ Health Impact Assessment Directorate, Provincial Health Data Centre, Western Cape Department of Health, Cape Town, South Africa
}

Corresponding author: P Raubenheimer (peter.raubenheimer@uct.ac.za)

Background. Globally there is increasing awareness of the need for end-of-life care and palliative care in hospitalised patients who are in their final year of life. Limited data are available on palliative care requirements in low- and middle-income countries, hindering the design and implementation of effective policies and health services for these patients.

Objectives. To determine the proportion of patients who die within 1 year of their date of admission to public hospitals in South Africa (SA), as a proxy for palliative care need in SA.

Methods. This was a retrospective cohort study using record linkage of admission and mortality data. The setting was 46 acute-care public hospitals in Western Cape Province, SA.

Results. Of 10761 patients (median (interquartile range (IQR)) age $44(31$ - 60) years) admitted to the 46 hospitals over a 2-week period in March 2012, 1570 (14.6\%) died within 1 year, the majority within the first 3 months. Mortality rose steeply with age. The median (IQR) age of death was 57.5 ( 45 - 70) years. A greater proportion of patients admitted to medical beds died within 1 year (21.3\%) compared with those admitted to surgical beds (7.7\%).

Conclusions. Despite a median age $<60$ years at admission, a substantial percentage of patients admitted to public sector hospitals in SA are in the final year of their lives. This finding should be seen in the context of SA's high communicable and non-communicable disease burden and resource-limited public health system, and highlights the need for policy development, planning and implementation of end-of-life and palliative care strategies for hospitals and patients.

S Afr Med J 2021;111(11):1070-1073. https://doi.org/10.7196/SAMJ.2021.v111i11.15869

Palliative care can be defined as an approach that improves the quality of life and provides relief of pain and suffering for patients and their families facing incurable, life-limiting disease. This holistic, patient-centred approach focuses not only on biomedical care but also on psychosocial and spiritual support. ${ }^{[1]}$ Globally, there is a growing need to provide appropriate population-level palliative care, as $\sim 58$ million people die annually, 45 million in low- and middleincome countries (LMICs). ${ }^{[1]}$ Data from high-income countries (HICs) provide estimates that $69-82 \%$ of people at the end of life will benefit from palliative care. ${ }^{[2]}$

It is important to understand population-level palliative care requirements in order to guide health service planning. The population need for palliative care has been estimated in several HICs using different approaches and methods. ${ }^{[3]}$ Mainstay approaches for assessing palliative care need include prospective predictive assessments based on patient diagnosis and assessment or retrospective analysis of 1-year post-admission death registry data. ${ }^{[3,4]}$

A dearth of evidence on population-based estimates of palliative care need in the sub-Saharan African health context hinders the effective development of service provision for terminal patients. ${ }^{[5]}$ A multicountry study conducted in Tanzania, Botswana, Ethiopia, Uganda and Zimbabwe found that at least $0.5 \%$ of the total population required palliative care. ${ }^{[6]}$ In South Africa (SA) there are no national data specifically compiled on palliative care need. Recent death statistics from SA describe annual deaths (2016) totalling $456612 .{ }^{[7]}$ Of these, the highest percentage $(7.8 \%)$ occurred in the age group 65 - 69 years. SA has a dual burden of disease, with leading causes of death in 2016 being attributed to both circulatory disorders (18.5\%) and infectious diseases (18.2\%). ${ }^{[7]}$ However, there is currently a strong national and provincial drive for palliative care in SA, and the National Health Council has approved a national health policy for palliative care ${ }^{[8,9]}$ Using only mortality data, it is estimated that $49.7 \%$ of people dying in SA would have benefited from palliative care. ${ }^{[8]}$

The role of hospitals and institutions in meeting the palliative care need of patients in SA is significant, as $43.2 \%$ of deaths occur in hospital, with $22.6 \%$ occurring at home. ${ }^{[7]}$ Early identification and timely care planning could therefore assist in more efficient utilisation of scarce health resources and enhanced quality of life in the end stage, coupled with a dignified death. To date, there are only two published studies that have estimated the prevalence of palliative care need in acute-care hospitals in the SA context. A prospective point-prevalence study of 11 hospitals in the Cape Town metro conducted in 2011, using a contextualised Gold Standards predictive tool, found a $16.6 \%$ prevalence of palliative care need among hospital inpatients. ${ }^{[10]} \mathrm{A}$ second study, retrospectively reviewing mortality data from a tertiary hospital general medicine service in Western 
Cape Province, determined mortality to be $11 \%$ at 3 months post discharge and $42 \%$ at 12 months post discharge. ${ }^{[1]}$ Using 1-year mortality as a palliative care needs estimate showed that nearly half of the patients admitted to general medical wards in this study could potentially have benefited from palliative care interventions. There is therefore a significant requirement for palliative care at hospital level, especially in medical wards.

\section{Objectives}

To develop a more comprehensive evidence base for the prevalence of palliative care need in SA. This study used routine health data to ascertain the 1-year mortality after hospital admission, using this as a proxy indicator for potential palliative care need of patients across all acute-care hospitals in the Western Cape.

\section{Methods}

\section{Participants and setting}

Health services in SA are provided by a mixture of public and private providers and institutions. Of an estimated 56.5 million people (2017) in SA, 84\% were dependent on government-funded health services. While it is estimated that SA spends $8.5 \%$ of its gross domestic product (GDP) on healthcare, a stark public-private divide is reflected in the fact that only $4.1 \%$ of the GDP is spent on the majority of people utilising public healthcare, while $4.4 \%$ is spent on $16 \%$ of the population accessing private healthcare. ${ }^{[12]}$ Alongside financial and human resource constraints in the public sector, care delivery is influenced by the high burden of disease and the low socioeconomic status of the majority of patients. ${ }^{[13]}$

In the Western Cape, $75 \%$ of the 5.8 million population utilise public health services. The province has 34 district hospitals with limited specialist services, 4 regional hospitals with some 24-hour specialist services, and 3 tertiary/quaternary referral health facilities (a total of 9802 inpatient hospital beds), delivering different levels of adult and child inpatient and outpatient care based on severity of need, and emergency, psychiatric, rehabilitative and obstetric services. There are also 4 dedicated psychiatric hospitals and 6 tuberculosis (TB) hospitals. ${ }^{[14]}$ Dedicated oncology inpatient services are only provided at the tertiary/quaternary hospitals.

For this study, 46 government hospitals in the Western Cape were sampled, including 31 district-level hospitals, 4 regional hospitals, 5 dedicated TB hospitals, 4 psychiatric hospitals and 2 tertiary/ quaternary hospitals. Hospitals providing only obstetric or paediatric services were excluded.

\section{Data sources}

Patients seeking inpatient and outpatient services at health facilities in the Western Cape are assigned a unique patient identification number (UPIN). This number is used to record all encounters across the different levels of care. The Western Cape Provincial Health Data Centre collects and links all electronic health data from individuals attending the public health service, including mortality data from hospital sources. ${ }^{[15]}$ For the time range of this study, mortality data were also available from death certification sources. Each UPIN was assigned a unique study-specific identification number.

\section{Study design}

A retrospective cohort study was conducted of all patients admitted to 46 acute-care hospitals in the Western Cape during a randomly selected 2-week period from 15 March to 29 March 2012. The year 2012 was selected because it had the most comprehensive mortality data.
Patients aged $\geq 18$ years on admission were included. Only the first admission during the time frame was included. All obstetric admissions were excluded. The final cohort consisted of 10761 patients. Data-set variables were age, sex, date of admission, date of discharge, the admitting department/specialty, and date of death.

Data management and statistical analysis were conducted in Stata version 13.1 (StataCorp, USA). Descriptive statistics were used to summarise patient data, namely frequency and percentage for categorical data and median with interquartile range (IQR) for continuous data. Ethics approval was granted by the University of Cape Town's Faculty of Health Human Research Ethics Committee (ref. no. 778/2016). Patient consent was not needed because the data were extracted from routinely collected data collated by the Western Cape Provincial Health Data Centre. Permission for use of the data set was obtained from the Data Centre on 18 November 2016, and the data were provided by the Provincial Health Impact Assessment Directorate.

\section{Results}

A total of 10761 patients met the study inclusion criteria during the 2-week study period. There were more women $(54.1 \%)$ than men $(45.9 \%)$. The median (IQR) age was $44(31-60)$ years, and the majority of the patients $(74.9 \%)$ were aged $<60$ years (Table 1 ).

There were 1570 deaths (14.6\%) that occurred within a year of the patient's date of admission. Four hundred patients (25.5\%) died during the index admission, while 1170 of the deaths occurred after discharge ( $74.5 \%$ of all deaths). Overall, $25.9 \%$ of the deaths occurred during the first 7 days $(n=406), 45.8 \%$ during the first 30 days $(n=719), 68.3 \%$ by 90 days $(n=1072)$ and $83.7 \%$ during the first 180 days $(n=1314)$. Mortality rose with age (Fig. 1$)$ and, as expected, was almost six times higher in patients aged $\geq 80$ years than in those aged $<30$ years $(34.7 \%$ v. $6.3 \%)$ at 1 year post admission.

Most patients were admitted to district hospitals ( $n=5795 ; 53.8 \%)$, followed by tertiary/quaternary hospitals $(n=2757 ; 25.6 \%)$ and then regional hospitals $(n=1945 ; 18.1 \%)$. Mortality did not differ significantly between the different categories of hospitals (district $14.7 \%$, regional $13.2 \%$, tertiary $15.7 \%$ ). Specialist psychiatric hospitals admitted 171 patients during the study period, of whom 5 (2.9\%) died during the year that followed admission. Specialist TB hospitals had the highest mortality; of 93 admissions, 27 patients (29.9\%) died within 1 year of admission.

Approximately half of the patients, $(n=5576 ; 51.8 \%)$ were admitted to medical specialties (internal medicine and oncology), while 4765 (44.2\%) were admitted to surgical specialties (general surgery, orthopaedics and gynaecology). Of all admissions to the medical specialties, 1130 patients (20.7\%) died within 1 year of admission; oncology admissions had the highest mortality rate of $50.8 \%$. Mortality was lower for patients admitted to surgical specialties $(9.2 \%$ for general surgery and $5.3 \%$ for orthopaedic surgery). Gynaecological mortality was only $2.7 \% 1$ year after admission.

\section{Discussion}

We established that $14.6 \%$ of all patients aged $>18$ years admitted to medical and surgical beds in public hospitals in the Western Cape died within 1 year of admission; 1 in 5 patients admitted to medical wards and almost 1 in 10 patients admitted to surgical wards died within the next year. The higher percentage of deaths in patients admitted to medical beds probably reflects the natural burden of disease in SA, with death mainly due to non-communicable diseases (ischaemic heart disease, stroke, diabetes) and communicable diseases (HIV and TB). ${ }^{[16]}$ It is also possible that admission to surgical beds is more likely to be elective in nature, whereas most 
Table 1. Characteristics of the patient cohort and mortality rates

\begin{tabular}{|c|c|c|c|c|c|c|c|}
\hline & \multicolumn{2}{|c|}{ All admissions } & \multicolumn{3}{|c|}{ Deaths within 1 year } & \multicolumn{2}{|c|}{ Alive after 1 year } \\
\hline & $n$ & $\%$ of total & $n$ & $\%$ of deaths & Mortality rate, \% & $n$ & $\%$ of alive \\
\hline Total & 10761 & 100.0 & 1570 & 100 & 14.6 & 9191 & 100.0 \\
\hline \multicolumn{8}{|l|}{ Gender } \\
\hline Male & 4944 & 45.9 & 774 & 49.3 & 15.7 & 4170 & 45.4 \\
\hline Female & 5817 & 54.1 & 796 & 50.7 & 13.7 & 5021 & 54.6 \\
\hline \multicolumn{8}{|l|}{ Age (years) } \\
\hline Median & 44 & & 57.5 & & & 42 & \\
\hline $18-29$ & 2358 & 21.9 & 94 & 6.0 & 4.0 & 2264 & 24.6 \\
\hline $30-39$ & 2033 & 18.9 & 183 & 11.7 & 9.0 & 1850 & 20.1 \\
\hline $40-49$ & 1925 & 17.9 & 236 & 15.0 & 12.3 & 1689 & 18.4 \\
\hline $50-59$ & 1745 & 16.2 & 321 & 20.4 & 18.4 & 1424 & 15.5 \\
\hline $60-69$ & 1355 & 12.6 & 328 & 20.9 & 24.2 & 1027 & 11.2 \\
\hline $70-79$ & 907 & 8.4 & 256 & 16.3 & 28.2 & 651 & 7.1 \\
\hline $80-89$ & 387 & 3.6 & 128 & 8.2 & 33.1 & 259 & 2.8 \\
\hline$\geq 90$ & 51 & 0.5 & 24 & 1.5 & 47.1 & 27 & 0.3 \\
\hline \multicolumn{8}{|l|}{ Specialties } \\
\hline Medicine & 5458 & 50.7 & 1130 & 72.0 & 20.7 & 4328 & 47.1 \\
\hline Surgery & 3393 & 31.5 & 312 & 19.9 & 9.2 & 3081 & 33.5 \\
\hline Gynaecology & 729 & 6.8 & 20 & 1.3 & 2.7 & 709 & 7.7 \\
\hline Orthopaedics & 643 & 6.0 & 34 & 2.2 & 5.3 & 609 & 6.6 \\
\hline Psychiatry & 354 & 3.3 & 10 & 0.6 & 2.8 & 344 & 3.7 \\
\hline Oncology & 118 & 1.1 & 60 & 3.8 & 50.8 & 58 & 0.6 \\
\hline Other & 66 & 0.6 & 4 & 0.3 & 6.1 & 62 & 0.7 \\
\hline \multicolumn{8}{|l|}{ Hospital type } \\
\hline District & 5795 & 53.8 & 850 & 54.2 & 14.7 & 3196 & 34.8 \\
\hline Tertiary/quaternary & 2757 & 25.6 & 432 & 27.5 & 15.7 & 2345 & 25.5 \\
\hline Regional & 1945 & 18.1 & 256 & 16.3 & 13.2 & 1689 & 18.4 \\
\hline Specialist psychiatry & 171 & 1.6 & 5 & 0.3 & 2.9 & 166 & 1.8 \\
\hline Specialist TB & 93 & 0.9 & 27 & 1.7 & 29.0 & 66 & 0.7 \\
\hline
\end{tabular}

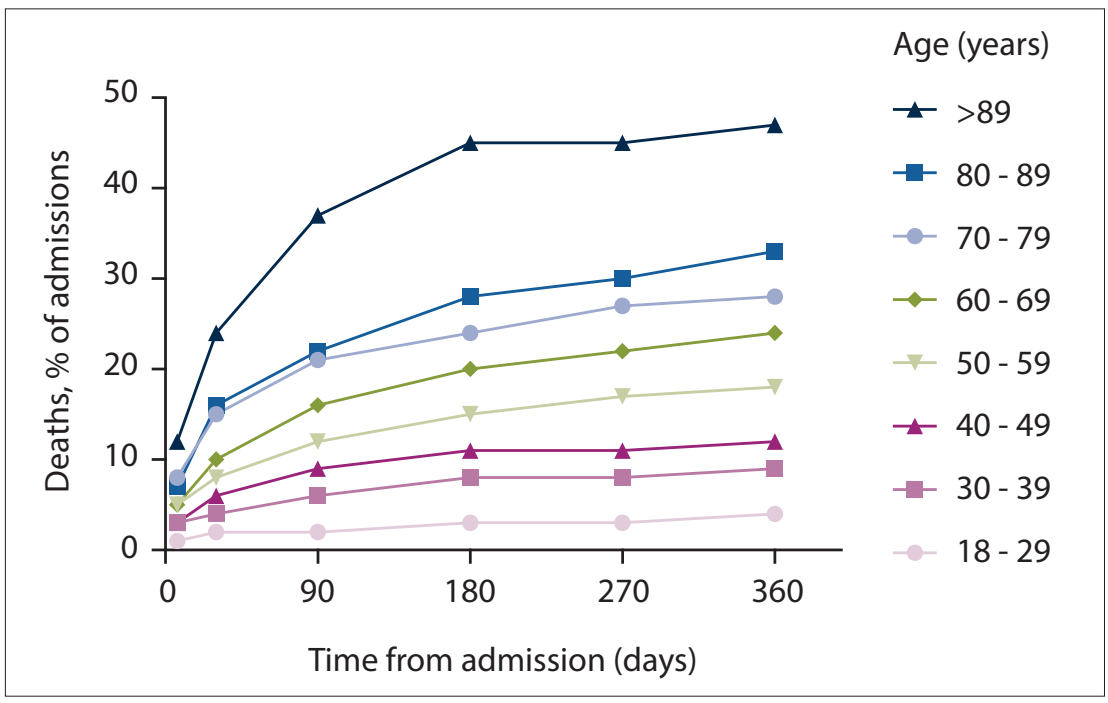

Fig. 1. Cumulative deaths as a percentage of census admissions, by age.

medical admissions are likely to be due to acute deterioration. Although the numbers were small, patients admitted to specialised oncology wards and those admitted to TB hospitals had very high 1-year mortalities of
$50.8 \%$ and $29.9 \%$, respectively. Overall the largest number of deaths were at districtlevel hospitals and hospitals with general specialist services only, probably reflecting that the greatest number of beds are available there, given that mortality rates between levels of hospitals were similar. Larger numbers of patients in their final year of life were admitted to medical wards, suggesting that the greatest need for palliative services would be in these wards at all hospital levels.

Our estimates of mortality 1 year after admission lie within a similar range to 12-month mortality rates in some HICs, for example $11.6 \%$ in Ontario, Canada, $14.5 \%$ in New Zealand, 22\% in Denmark, $22.4 \%$ in Scotland, and $>25 \%$ in a subset of patients aged $>65$ years in Chicago, USA. ${ }^{[17-21]}$ The median age of admission in these countries was higher than in our SA cohort, possibly owing to larger elderly populations in those countries and the dual burden of communicable and non-communicable diseases in SA resulting in a relatively younger population. All studies also showed a strong correlation with mortality and age, as expected. The present study is the first study using record linkage data to estimate 1-year mortality as a proxy for palliative care requirements in an African and LMIC context. 


\section{Study strengths and limitations}

This is the first study in Africa to use data linkage and a unique patient identification number to link hospital admission data with mortality records and use this information as a proxy to estimate palliative care requirements. The study population is therefore different to those of HICs, with a younger age of admission reflecting the demographic transition of non-communicable diseases seen in LMICs, as well as a higher HIV and TB burden of disease. These findings may not be directly translatable to other provinces in SA, because the Western Cape has a lower burden of HIV disease and better access to healthcare than other areas in SA. ${ }^{[2]}$ The study is also limited in that data were only available for patients who died in the Western Cape; mortality of patients who moved to another province may not have been captured. As such, it can still provide a conservative estimate that may under-report total mortality. Given the incomplete ICD-10 coding for admission and on death certification, we were unable to estimate how many deaths were unrelated to the initial hospital admission, for example a death due to an unnatural cause, which may have biased our extrapolation to palliative care requirements towards an overestimate. Using mortality data is only a surrogate for the number of patients in the last year of their life, and does not directly speak to actual palliative care need of patients, which should be provided alongside curative care and earlier in the disease course, as required. Palliative care may of course also be required by patients who live longer than a year.

\section{Conclusions}

At least 1 in 7 patients admitted to public hospitals in the Western Cape will die within the next year. Admission to hospital therefore provides an important opportunity to attempt to identify these patients and where appropriate to provide palliation and initiate discussions around end-of-life care, advanced care planning and palliative care. Most need would be in the medical specialties, as the largest proportion of patients who are in their final year of life are in these wards.

Declaration. The research for this study was done in partial fulfilment of the requirements for PF's MMed (Int Med) degree at the University of Cape Town.

Acknowledgements. Provincial Health Data Centre of the Western Cape. Author contributions. PF and PJR designed and led the study. PF, PJR and LvN designed and wrote the study protocol. NT extracted the data set from the Western Cape Provincial Health Data Centre. PF, PJR, KM and NT analysed and interpreted the data. PF wrote the first draft of the report, and all authors reviewed and approved the final version.
Funding. None.

Conflicts of interest. None.

1. Stjernsward J, Foley KM, Ferris FD. Integrating palliative care into national policies. J Pain Symptom Manage 2007;33(5):514-520. https://doi.org/10.1016/j.jpainsymman.2007.02.031

2. Murtagh FE, Bausewein C, Verne J, Groeneveld EI, Kaloki YE, Higginson IJ. How many people need palliative care? A study developing and comparing methods for population-based estimates. Palliat

3. Gardiner C, Gott M, Ingleton C, et al. Extent of palliative care need in the acute hospital setting: A survey of two acute hospitals in the UK. Palliat Med 2013;27(1):76-83. https://doi. org/10.1177/0269216312447592

4. Gott M, Frey R, Raphael D, O'Callaghan A, Robinson J, Boyd M. Palliative care need and management in the acute hospital setting: A census of one New Zealand hospital. BMC Palliat Care 2013;12:15. https://doi.org/10.1186/1472-684X-12-15

5. Harding R, Powell RA, Doowning J, Garanganga E, Kikule E, Alexander C. Generating an African palliative care evidence base: The context, need, challenges, and strategies. J Pain Symptom Manag 2008;36(3):304-309. https://doi.org/10.1016/j.jpainsymman.2008.04.008

6. Sepulveda C, Habiyambere V, Amandua J, et al. Quality care at the end of life in Africa. BMJ 2003;327:209. https://doi.org/10.1136/bmj.327.7408.209

7. Statistics South Africa. Mortality and causes of death in South Africa, 2016: Findings from death notification. Statistical release P0309.3. Pretoria: Stats SA, 27 March 2018. https://www.statssa.gov.za/ publications/P03093/P030932016.pdf (accessed 29 July 2021)

8. Department of Health, South Africa. National Policy Framework and Strategy on Palliative 8. Department of Health, South Africa. National Policy Framework and Strategy on Palliative
Care 2017 - 2022. Pretoria: DoH, 2017. http://www.health.gov.za/wp-content/uploads/2020/11/ NationalPolicyFrameworkandStrategyonPalliativeCare20172022.pdf (accessed 29 July 2021).

9. GationalPolicyFrameworkandStrategyonPalliativeCare 20172022 .pdf (accessed 29 July 2021 ).
Gwyther LC, Stanford C, Grey J, et al. The development of hospital-based palliative care services in public hospitals in the Western Cape, South Africa. S Afr Med J 2018;108(2):86-89. https://doi. org/10.7196/SAMJ.2017.v108i2.12524

10. Van Niekerk L, Raubenheimer PJ. A point-prevalence survey of public hospital inpatients with palliative care needs in Cape Town, South Africa. S Afr Med J 2013;104(2):138-141. https://doi. org/10.7196/SAMJ.7262

11. Stuart-Clark H, Vorajee N, Zuma S, et al. Twelve-month outcomes of patients admitted to the acute general medical service at Groote Schuur Hospital. S Afr Med J 2012;102(6):549-553. https://doi. org/10.7196/SAMJ.5615

12. Department of Health, South Africa. National Health Insurance for South Africa: Towards universal health coverage. Pretoria: DoH, 2017. https://www.gov.za/sites/default/files/gcis_ document/201707/40955gon627.pdf (accessed 29 July 2021)

13. Mayosi BM, Benatar SR. Health and health care in South Africa - 20 years after Mandela. N Engl J Med Mayosi BM, Benatar SR. Health and health care in South Africa

4. Western Cape Government: Health. Annual Report 2015/16. 2016. https://www westerncape.gov.za/ Western Cape Government: Health. Annual Report 2015/16. 2016. https://ww).wcts/departments/health/annual_report_2015_16.pdf (accessed 29 July 2021)

assets/departments/health/annual_report_2015_16.pdf (accessed 29 July 2021).
5. Boulle A, Heekes A, Tiffin N, et al. Data centre profile: The Provincial Health Data Centre of the Western Cape Province, South Africa. Int J Popul Data Sci 2019;4(2). https://doi.org/10.23889/ijpds. v4i2.1143

16. Statistics South Africa. Mortality and causes of death in South Africa, 2013: Findings from death notification. Statistical release P0309.3. Pretoria: Stats SA, 2 December 2015. https://www.statssa.gov. za/publications/P03093/P030932014.pdf (accessed 29 July 2021).

17. Flojstrup M, Henriksen DP, Brabrand M. An acute hospital admission greatly increases one year mortality - Getting sick and ending up in hospital is bad for you: A multicentre retrospective cohort study. Eur J Intern Med 2017;45:5-7. https://doi.org/10.1016/i.ejim.2017.09.035

18. Clark D, Armstrong M, Allan A, Graham F, Carnon A, Isles C. Imminence of death among hospital inpatients: Prevalent cohort study. Palliat Med 2014;28(6):474-479. https://doi. org/10.1177/0269216314526443

19. Levine SK, Sachs GA, Jin L, Meltzer D. A prognostic model for 1-year mortality in older adults after hospital discharge. Am J Med 2007;120(5):455-460. https://doi.org/10.1016/j.amjmed.2006.09.021

20. Van Walraven C. Trends in 1-year survival of people admitted to hospital in Ontario, 1994 - 2009. Can . Van Walraven C. Trends in 1-year survival of people admitted to hospital in
Med Assoc J 2013;185(16):E755-E762. https://doi.org/10.1503/cmaj.130875

21. Gott M, Broad J, Zhang X, Jarlbaek L, Clark D. Likelihood of death among hospital inpatients in New Zealand: Prevalent cohort study. BMJ Open 2017;7:e016880. https://doi.org/10.1136/ bmjopen-2017-016880

22. Human Sciences Research Council. The fifth South African national HIV prevalence, incidence, behaviour and communication survey. July 2018. http://www.hsrc.ac.za/uploads/ pageContent/9234/SABSSMV_Impact_Assessment_Summary_ZA_ADS_cleared_PDFA4.pdf (accessed 29 July 2021)

Accepted 30 June 2021. 